\title{
Symmetric Sum-Free Partitions and Lower Bounds for Schur Numbers
}

\author{
Harold Fredricksen \\ Naval Postgraduate School, \\ Department of Mathematics, \\ Monterey, CA 93943, USA \\ halF@nps . navy.mil
}

\author{
Melvin M. Sweet \\ Institute for Defense Analyses, \\ Center for Communications Research, \\ 4320 Westerra Court, \\ San Diego, CA 92121, USA \\ sweet@ccrwest.org
}

Submitted:May 9, 2000; Accepted:May 18, 2000

\begin{abstract}
We give new lower bounds for the Schur numbers $S(6)$ and $S(7)$. This will imply new lower bounds for the Multicolor Ramsey Numbers $R_{6}(3)$ and $R_{7}(3)$. We also make several observations concerning symmetric sumfree partitions into 5 sets.
\end{abstract}

\section{Introduction}

A set of integers is said to be sum-free if for all $i$ and $j \geq i$ in the set the sum $i+j$ is not in the set. The Schur number $S(k)$ is defined to be the maximum integer $n$ for which the interval $[1, n]$ can be partitioned into $k$ sum-free sets. ${ }^{1}$ I. Schur [7] proved that $S(k)$ is finite, and that

$$
S(k) \geq 3 S(k-1)+1 .
$$

In the framework of Ramsey theory, Schur's proof yields the inequality

$$
S(k) \leq R_{k}(3)-2,
$$

where $R_{k}(n)$ denotes the $k$-color Ramsey number, defined to be the smallest integer such that any $k$-color edge coloring of the complete graph on $R_{k}(n)$ vertices has at least one complete subgraph all of whose edges have the same color.

\footnotetext{
${ }^{1}$ Some authors define $S(k)$ to be the smallest $n$ for which there are no sum-free partitions into $k$ sets.
} 
It is easily verified that $S(1)=1, S(2)=4$, and $S(3)=13$; and L.D. Baumert [1] showed in 1961 with the aid of a computer that $S(4)=44$. The best known bounds for $S(5)$ are

$$
160 \leq S(5) \leq 315
$$

The first inequality in (3) is due to G. Exoo [4], and the second follows from (2) and the bounds for $R_{5}(3)$ given in S. Radziszowski's survey paper [6]. For earlier work on lower bounds for $S(k)$ see H. Fredricksen [5] and A. Beutelspacher and W. Brestovansky [2].

The best previously known lower bound $S(6) \geq 481$ for $S(6)$ follows from (1). At the end of this note we list constructions that show

$$
\begin{aligned}
& S(6) \geq 536 \\
& S(7) \geq 1680 .
\end{aligned}
$$

Using (2) we obtain the following lower bounds for the Ramsey numbers $R_{6}(3)$ and $R_{7}(3)$ :

$$
\begin{aligned}
& R_{6}(3) \geq 538 \\
& R_{7}(3) \geq 1682 .
\end{aligned}
$$

This improves the bounds given in Radziszowski's survey paper. Note that the bound for $R_{7}(3)$ is better than the lower bound of 1662 that can be obtained by using F. Chung's [3] inequality

$$
R_{k+1}(3) \geq 3 R_{k}(3)+R_{k-2}(3)-3, \quad \text { for } \quad k \geq 3,
$$

with our new bound for $R_{6}(3)$.

The constructions and results in this paper focus on symmetric partitions, where a sum-free partition $P$ of the interval $[1, n]$ is said to be symmetric if all symmetric pairs $i$ and $(n+1)-i$ are in the same set; except in the case when $n+1$ is divisible by 3 , we must allow $(n+1) / 3$ and $2(n+1) / 3$ to be in different sets. There are no real theorems in this note, only observations and conjectures based on a computer study of symmetric sum-free partitions. We have restricted our focus to such partitions because the search space is smaller, and the concepts of equivalence and depth which are explained below provide additional useful structure for restricting the search.

It is easy to see that if a symmetric sum-free partition $P$ is multiplied $\bmod (n+1)$ by an integer $m$ that is relatively prime to $n+1$, then $m P$ is also a symmetric sum-free partition. We will say two such partitions are equivalent.

Given a partition $P$ of $[1, n]$, we define the depth, denoted $d(P)$, to be the largest of the set minimums; and for a symmetric sum-free partition we define the e-depth, denoted $D(P)$, to be the maximum of $d\left(P^{\prime}\right)$ over all partitions $P^{\prime}$ equivalent to $P$. We define $D_{k}(n)$ to be the maximum of $D(P)$ over all symmetric sum-free partitions $P$ of $[1, n]$ into $k$ sets. Note that we must have

$$
D_{k}(n) \leq S(k-1)+1 \text {. }
$$


In a later section we will give exact values of $D_{5}(n)$ for many values of $n$. These values and other studies described later for partitions into 5 sets have led us to make the following conjectures:

Conjecture 1 The largest integer for which there is a symmetric sum-free partition into 5 sets is 160; and perhaps $S(5)=160$.

Conjecture 2 All symmetric sum-free partitions of 160 into 5 sets have e-depth 44.

Conjecture 3 There are no symmetric sum-free partitions of 155 or 158 into 5 sets.

Note 155 and 158 are the special cases in the definition of symmetric, and that it is not obvious that a symmetric partition of $n$ into $k$ sets implies that there is a symmetric partition of $m$ into $k$ sets for $m<n$.

\section{The Search Algorithm}

The search algorithm we used is the obvious branching scheme modified with various heuristics. We successively place an integer that is "most blocked" (in set membership) by earlier placements, and then backtrack when a branch dies or an answer is found. The heuristic used to decide which of the most blocked integers to select was to choose the smallest. Choosing the correct heuristic can make a huge difference in search time. Using this algorithm with the condition $d(P) \geq m$, it is possible to completely exhaust the search tree in less than a minute for partitions of $[1, n]$ into 5 sets with $n$ near 160 and $m$ near to $44=S(4)$. For smaller depths the running time required to exhaust becomes excessive; but by limiting the total number of placements made beyond a certain level it is possible to probabilistically prune the search tree and have a good probability of finding an answer when one exists.

Our search algorithm and heuristics were motivated by the following three observations that evolved during the development of the algorithm:

1. It is easy to exhaustively find all (not necessarily symmetric) sum-free partitions into 4 sets.

2. With high probability, it is easy to complete in a few steps a symmetric sum-free partition if only about $20 \%$ of the elements are known.

3. Many partitions are equivalent to partitions with large depth which can be build by first finding partitions in fewer sets.

The partitions $\mathrm{P}$ of $k=6$ and 7 sets given at the end of this paper were found using a probabilistic algorithm starting with a partition $P^{\prime}$ of $k-1$ sets with small $d\left(P^{\prime}\right)$ and by restricting $d(P)$ to be near $S(k-1)$. 
We believe that it should be possible to find a larger lower bound for $S(6)$ by looking for symmetric sum-free partitions that have large depth and have one other set with large e-depth. We currently have programs running to do this.

\section{Conjectures and Observations for 5 Sets}

This section describes observations and conjections resulting from a computer study of symmetric sum-free partitions into 5 sets.

By an exhaustive branching program, we were able to determine $D_{5}(n)$ for all $n \leq 154$. For $102 \leq n \leq 154, D_{5}(n)$ is always either 44 or 45 ; for $86 \leq n \leq 101$, $D_{5}(n)$ is between 41 and 45 ; and for $45 \leq n \leq 85, D_{5}(n)=[(n+1) / 2]$. We were also able to determine that $D_{5}(160)=D_{5}(157)=D_{5}(156)=44$, but that $D_{5}(159)=39$. We were only able to show exhaustively that $D_{5}(155)<36$ and that $D_{5}(158)<36$. A probabilistic search for symmetric sum-free partitions of 158 into 5 parts, leads us to conjecture that there may be no such partitions; it is not obvious that a symmetric partition of $n$ into $k$ sets implies that there is a symmetric partition of $m$ into $k$ sets for $m<n$. A similar statement may apply to 155 , but we have not examined it as carefully. For $160<n \leq 315$ we exhaustively searched for symmetric partitions into 5 sets with depth $\geq 44$ and found that there are none. We did the same for other more relaxed depth restrictions, but for smaller $n$, with the same result; to summarize

$$
\begin{aligned}
& D_{5}(n)<36 \quad \text { for } 161 \leq n \leq 190 \\
& D_{5}(n)<39 \quad \text { for } 191 \leq n \leq 210 \\
& D_{5}(n)<44 \quad \text { for } 211 \leq n \leq 315
\end{aligned}
$$

This, various probabilistic searches, and the upper bound in (3) leads us to conjecture that 160 is the largest integer for which there is a symmetric sumfree partition into 5 sets, and perhaps that $S(5)=160$.

We found that there are symmetric sum-free partitions of 157 into 5 sets for each of the e-depths 44,31, and 26. For partitions of 159 into 5 sets, we found examples with $D(P)=39$ and examples with $D(P)=26$. The only e-depth found for partitions of 160 into 5 sets was 44 . We conjecture that no other e-depths are possible for 160 into 5 sets. This would mean that all partitions of 160's would be equivalent to the 5840 we found exhaustively for 160 with $d=44$; of these, 768 are equivalent to exactly one other partition with $d(P)=44$ (interestingly, the equivalence multiplier is always 30 or 59 $(=-1 / 30 \bmod 161))$, the rest were equivalent to no others with $d(P)=44$. So we conjecture that there are $(4304+(768 / 2)) * \phi(161) / 2=309,408$ symmetric sum-free partitions of 160 into 5 sets.

An interesting note for depth 44 symmetric sum-free partitions of 160 into 5 sets is that the shortest distance between one and a multiple of another is small. Here the distance between partitions is defined as the smallest number of elements that are in different sets between the partitions, after a relabeling of 
sets for one of the partitions. Considering only multiplication by 1, 30, and 59 the maximum distance is 19 . This means that there is essentially only one cluster of depth 44 partitions, and if our conjecture is correct, then every symmetric partition is equivalent to something in this cluster.

The situation in the last paragraph for 160 is similar to what happens for the largest partitions into 4 and 3 sets; further evidence that 160 may be maximal for 5 set partitions. For partitions of 44 into 4 sets all $24=\phi(45)$ symmetric sum-free partitions of the 273 sum-free partitions are equivalent (note that, since 3 divides 45 , multiplying by 1 and -1 are not the same), and 6 of the 24 have depth 13. For sum-free partitions of 13 into 3 sets all three partitions are symmetric and equivalent.

Another interesting aside, for depth 44 symmetric sum-free partitions of 160 into 5 sets is that the sizes of the sets have the following properties:

1. There are 128 different set size configurations.

2. The largest set size is 44 , and the smallest is 24 .

3. The largest difference between smallest and largest set in a partition is 20, and the smallest is 6 . For the largest difference, the largest set is always 44. For the smallest difference, the largest set is always 34 .

4. The most likely set size configuration is $\{26,28,32,34,40\}$, 
THE ELECTRONIC JOURNAL OF COMBINATORICS 7 (2000), \#R32

\section{Constructions}

The constructions exhibited below are symmetric, and so we only list the smallest of a symmetric pair.

Partition of 536 into 6 symmetric sumfree sets:

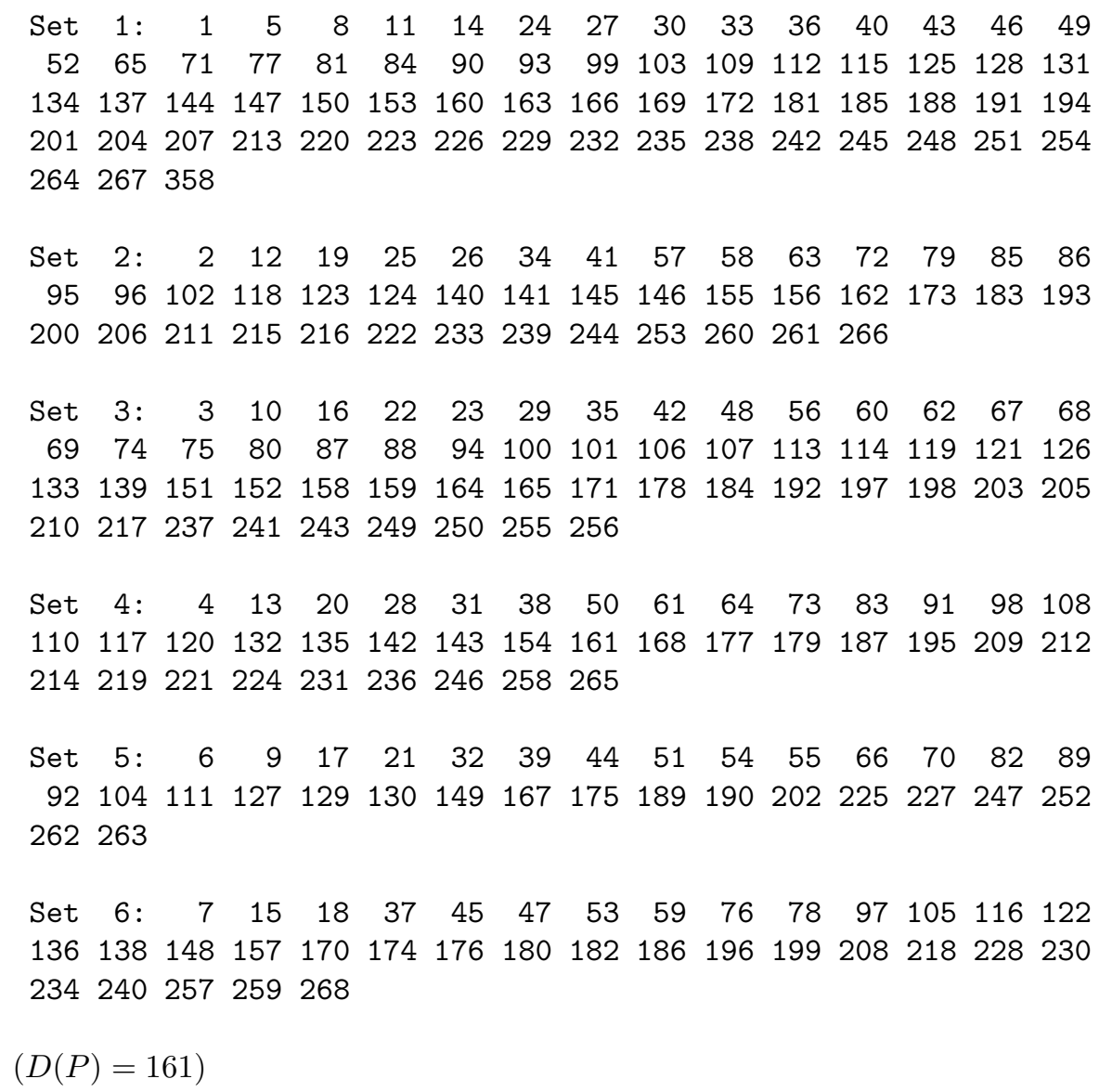

We also have constructions of 6 set partitions for the following $n$ and respective e-depths $D$ :

$n=499,506,510,511,512,513,514,515,516,517,518,519,520,523,525,526,531$

$D=160,161,160,161,161,161,144,147,158,144,142,161,161,161,155,160,161$. 
THE ELECTRONIC JOURNAL OF COMBINATORICS 7 (2000), \#R32

Partition of 1680 into 7 symmetric sumfree sets:

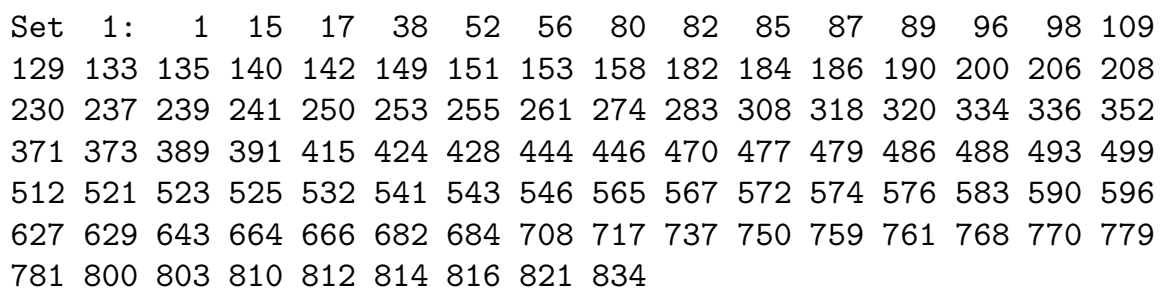




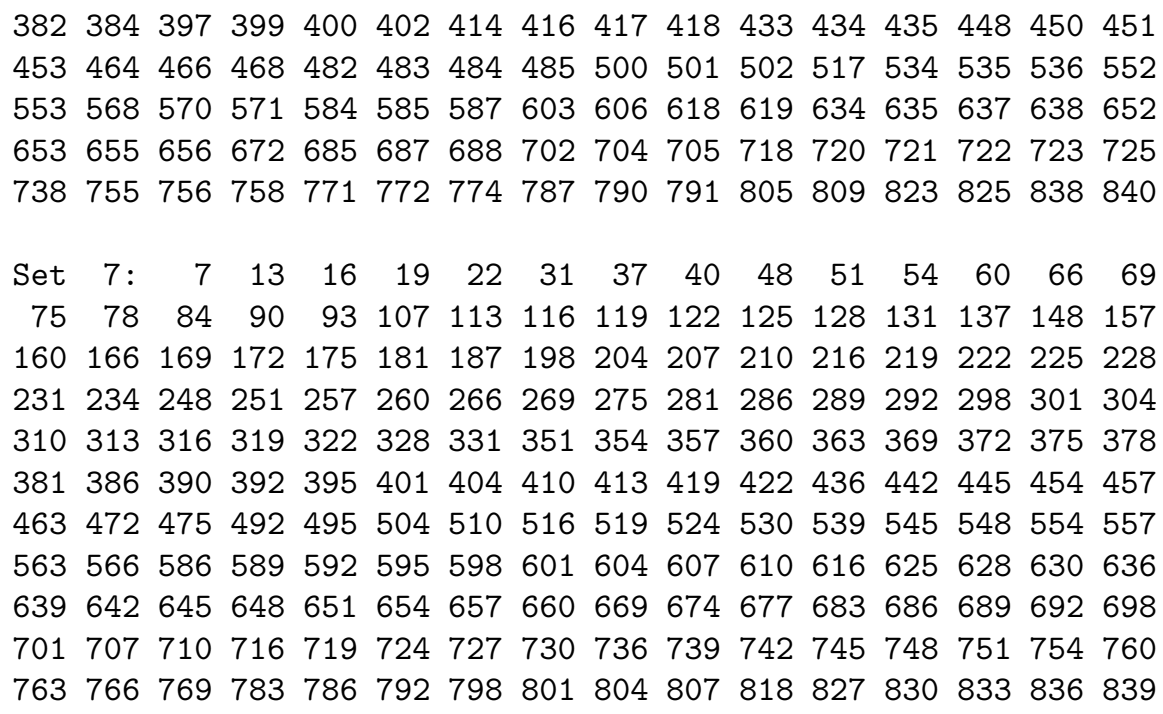

We also have constructions of 7 set partitions for

$$
\begin{aligned}
& n=1615, \quad 1620, \quad 1630, \quad 1640, \quad 1650, \quad 1660, \quad 1665 \\
& D=\quad 532, \quad 537, \quad 536, \quad 537, \quad 537, \quad 537, \quad 537 .
\end{aligned}
$$

\section{References}

[1] L. D. Baumert and S.W. Golomb. Backtrack Programming. J. Ass. Comp. Machinery, 12:516-524, 1965.

[2] Albrecht Beutelspacher and Walter Brestovansky. Generalized Schur Numbers. In Combinatorial Theory, Proceedings of a Conference Held at Schloss Rauischholzhausen, May 6-9, 1982, volume 969 of Lecture Notes in Mathematics, pages 30-38. Springer-Verlag, Berlin-New York, 1982.

[3] Fan Rong K. Chung. On the Ramsey Numbers $N(3,3, \ldots 3 ; 2)$. Discrete Mathematics, 5:317-321, 1973.

[4] Geoffrey Exoo. A Lower Bound for Schur Numbers and Multicolor Ramsey Numbers of $K_{3}$. The Electonic Journal of Combinatorics, http://www.combinatorics.org/, \#R8, 1: 3 pages, 1994.

[5] Harold Fredricksen. Schur Numbers and the Ramsey Numbers $N(3,3, \ldots, 3 ; 2)$. J. Combinatorial Theory Ser A, 27:371-379, 1979. 
[6] Stainislaw P. Radziszowski. Small Ramsey Numbers. Dynamic Survey DS1. Electronic J. Combinatorics, 1:35 pages, 1984 Revision \#6 July 5, 1999.

[7] I. Schur. Uber die Kongruenz $x^{m}+y^{m} \equiv z^{m}(\bmod p)$. Jahresber. Deutsch. Math.-Verin., 25:114-116, 1916. 